\title{
Nuevos desafíos a nivel latinoamericano para la Sociedad Chilena de Gastroenterología
}

\author{
New challenges at a Latin American level for the Chilean Society \\ of Gastroenterology
}

Este 2021, la Sociedad Chilena de Gastroenterología (SChGE) enfrenta nuevos desafíos ya que en el contexto de la pandemia por SARS CoV-2, recibimos con gran alegría la gestión de la Organización Panamericana de Gastroenterología (OPGE) y de la Sociedad Interamericana de Endoscopía Digestiva (SIED). Durante el mes de julio de 2021, se realizó la transmisión de mando desde Uruguay, asumiendo el Dr. Arnoldo Riquelme, la presidencia de OPGE por el periodo 2021-2023. A fin de año, el Dr. Fernando Fluxá recibirá la presidencia de SIED por el mismo período y esperamos desarrollar y expandir las actividades de extensión, educación continua y de investigación de ambas sociedades, promoviendo la integración de las Sociedades Científicas del continente con un especial énfasis en la globalización de la gastroenterología y hepatología, favoreciendo las interacciones con la Asociación Española de Gastroenterología (AEG), American College of Gastroenterology (AGA), American College of Gastroenterology (ACG), Asociación Latinoamericana para el estudio del Hígado (ALEH), United European Gastroenterology (UEG), World Gastroenterology Organization (WGO) y la World Endoscopy Organization (WEO). El contexto de la pandemia COVID19 confiere a nuestra gestión, múltiples desafíos incluyendo las dificultades relacionadas con la ejecución de actividades presenciales, pero son múltiples las experiencias exitosas de las actividades que desde la SChGE, hemos podido generar en pandemia, potenciando las actividades virtuales y aumentando nuestra globalización, con la participación de disertantes y asistentes conectados de manera remota desde los distintos rincones de los países hispano-parlantes. Esperamos que la transición gradual de la virtualidad a las actividades presenciales, nos permitan ofrecer a la región, un reencuentro en Santiago de Chile, el 2023, cerrando nuestra gestión conjunta para OPGE y SIED, de las Semanas Panamericanas de Gastroenterología (SPED). Esperamos los Dres. Fluxá y Riquelme, así como el comité ejecutivo de SPED, contar con la activa participación de los socios de la SChGE así como de las Asociaciones y filiales, para ofrecer al continente actividades educacionales y formativas diversas y frecuentes en el periodo 2021-2023.

La Revista Gastroenterología Latinoamericana seguirá siendo el órgano oficial de la SChGE, pero tendremos un especial énfasis en la apertura a las Sociedades Científicas de la región, para divulgar conocimiento nuevo así como revisiones o consensos de tópicos prevalentes en Iberoamérica.

En relación a las secciones de la revista, Diego Ruedi Z., Alexandros Hoyos B., Luis Méndez A. nos traen una interesante revisión titulada "Edulcorantes artificiales: ¿Dulce compañía?”. Los Dres. Gonzalo Latorre, Jaime Gómez, Jorge Arnold, Felipe Silva, Felipe Bellolio, Jorge Escarate, Manuel Álvarez, Alberto Espino y José Ignacio Vargas, presentan un interesante caso clínico de divertículo de Meckel. Por su parte, los Dres. Juan Orosco A., Cynthia Orrego C., César Rivas T. presentan el artículo Pseudomelanosis duodeni: a propósito de un caso. Revisión de la literatura.

La ya tradicional Sección "Gastro y algo más” recibe un sentido artículo de parte del_Dr. Roque Sáenz F. con el "In memoriam: Dr. Francisco Vilardell Viñas". En la misma sección, los Dres. Raimundo Izquierdo, Luis Pastén, Rodney Stock, Alex Navarro y Roque Sáenz nos transportan en un recorrido histórico de la endoscopía con el artículo "Desde la Clasificación de Kudo a la Inteligencia Artificial en Endoscopía. Una historia de 30 años. " Esta sección acoge con gran orgullo la reseña del Dr. José Luis Chianale B. sobre un nuevo premio Nacional de Medicina para la Gastroenterología, que el 2020 fue otorgado al Dr. Vicente Valdivieso Dávila.

La Sección "Medicina basada en la evidencia en Gastroenterología" nos entrega el análisis crítico de 2 estudios._Rodrigo Arias Z., Vicente Gándara F., María Francisca Seydewitz O., Francisco Tortorolo C., Carlos Benítez G. analizan el artículo “Atezolizumab y bevacizumab en carcinoma hepatocelular irresecable” y Magdalena Ruiz-Esquide S., Catalina Reyes P., Javier Rodríguez G., Luis Antonio Díaz P., Arnoldo Riquelme P., Fernando Pimentel M. "Colangiopancreatografía retrógrada endoscópica con papilotomía de urgencia 


\section{Editorial}

versus tratamiento conservador en pancreatitis aguda grave por cálculos biliares (APEC trial): un estudio aleatorizado multicéntrico".

Esperamos desde el comité editorial de la Revista Gastroenterología Latinoamericana, que este número sea del agrado de nuestros lectores y ofrecemos esta plataforma para la difusión científica de los investigadores clínicos del continente, para democratizar el conocimiento de nuestra disciplina.

Dr. Arnoldo Riquelme P.

Editor

Revista Gastroenterología Latinoamericana 